\title{
Buprenorphine transdermal system for opioid therapy in patients with chronic low back pain
}

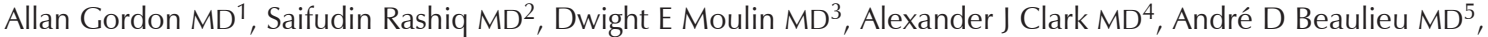 \\ John Eisenhoffer $\mathrm{MD}^{6}$, Paula S Piraino $\mathrm{PhD}^{6}$, Patricia Quigley $\mathrm{MSc}^{7}$, Zoltan Harsanyi $\mathrm{MBA}^{6}$, Andrew C Darke $\mathrm{PhD}^{6}$
}

A Gordon, S Rashiq, DE Moulin, et al. Buprenorphine transdermal system for opioid therapy in patients with chronic low back pain. Pain Res Manage 2010;15(3):169-178.

OBJECTIVE: The present randomized, double-blinded, crossover study compared the efficacy and safety of a seven-day buprenorphine transdermal system (BTDS) and placebo in patients with low back pain of moderate or greater severity for at least six weeks.

METHODS: Prestudy analgesics were discontinued the evening before random assignment to $5 \mu \mathrm{g} / \mathrm{h}$ BTDS or placebo, with acetaminophen $300 \mathrm{mg} /$ codeine $30 \mathrm{mg}$, one to two tablets every $4 \mathrm{~h}$ to $6 \mathrm{~h}$ as needed, for rescue analgesia. The dose was titrated to effect weekly, if tolerated, to $10 \mu \mathrm{g} / \mathrm{h}$ and $20 \mu \mathrm{g} / \mathrm{h} \mathrm{BTDS}$. Each treatment phase was four weeks.

RESULTS: Fifty-three patients ( 28 men, 25 women, mean $[ \pm$ SD] age $54.5 \pm 12.7$ years) were evaluable for efficacy (completed two weeks or more in each phase). Baseline pain was $62.1 \pm 15.5 \mathrm{~mm}$ (100 mm visual analogue scale) and $2.5 \pm 0.6$ (five-point ordinal scale). BTDS resulted in lower mean daily pain scores than in the placebo group $(37.6 \pm 20.7 \mathrm{~mm}$ versus $43.6 \pm 21.2 \mathrm{~mm}$ on a visual analogue scale, $\mathrm{P}=0.0487$; and $1.7 \pm 0.6$ versus $2.0 \pm 0.7$ on the ordinal scale, $\mathrm{P}=0.0358$ ). Most patients titrated to the highest dose of BTDS (59\% $20 \mu \mathrm{g} / \mathrm{h}, 31 \% 10 \mu \mathrm{g} / \mathrm{h}$ and $10 \% 5 \mu \mathrm{g} / \mathrm{h})$. There were improvements from baseline in pain and disability (Pain Disability Index), Pain and Sleep (visual analogue scale), Quebec Back Pain Disability Scale and Short-Form 36 Health Survey scores for both BTDS and placebo groups, without significant differences between treatments. While there were more opioid-related side effects with BTDS treatment than with placebo, there were no serious adverse events. A total of $82 \%$ of patients chose to continue BTDS in a long-term open-label evaluation, in whom improvements in pain intensity, functionality and quality of life were sustained for up to six months without analgesic tolerance. CONCLUSION: BTDS $(5 \mu \mathrm{g} / \mathrm{h}$ to $20 \mu \mathrm{g} / \mathrm{h})$ represents a new treatment option for initial opioid therapy in patients with chronic low back pain.

Key Words: Buprenorphine; Low back pain; Transdermal system

\section{Système transdermique de buprénorphine pour le traitement opiacé de la lombalgie basse chronique}

OBJECTIF : La présente étude randomisée, à double insu, avec permutation des groupes, comparait l'efficacité et l'innocuité d'un système transdermique de buprénorphine (STDB) de sept jours et d'un placebo chez des patients atteints de lombalgie basse d'intensité modérée ou plus, sur une période d'au moins six semaines.

MÉTHODES : Les analgésiques utilisés avant le début de l'étude ont été cessés la veille de la répartition aléatoire soit à $5 \mu \mathrm{g} / \mathrm{h}$ de STDB ou au placebo, avec acétaminophène $300 \mathrm{mg} /$ codéine $30 \mathrm{mg}$ à raison d'un ou deux comprimés toutes les quatre à six heures au besoin pour analgésie d'appoint. Selon l'effet obtenu, toutes les semaines, il était possible de porter la dose à $10 \mu \mathrm{g} / \mathrm{h}$ et $20 \mu \mathrm{g} / \mathrm{h}$ de STDB, si elle était tolérée. Chaque phase du traitement était d'une durée de quatre semaines.

RÉSULTATS : Cinquante-trois patients (28 hommes, 25 femmes, âge moyen [ \pm É.-T.] 54,5 $\pm 12,7$ ans) étaient évaluables sur le plan de l'efficacité (c.-à-d., avaient achevé deux semaines ou plus de chaque phase). La douleur au départ se situait à $62,1 \pm 15,5 \mathrm{~mm}$ (sur une échelle analogique visuelle de $100 \mathrm{~mm}$ et à $2,5 \pm 0,6$ (sur une échelle numérique en cinq points). Le STDB a donné lieu à une baisse plus marquée des indices douloureux quotidiens moyens vs placebo $(37,6 \pm 20,7 \mathrm{~mm}$ vs $43,6 \pm$ $21,2 \mathrm{~mm}$ à l'échelle analogique visuelle, $\mathrm{p}=0,0487$ et $1,7 \pm 0,6$ vs $2,0 \pm$ 0,7 à l'échelle numérique, $\mathrm{p}=0,0358$ ). La dose de STDB de la plupart des patients a été portée au maximum (59\%: $20 \mu \mathrm{g} / \mathrm{h}, 31 \%: 10 \mu \mathrm{g} / \mathrm{h}$ et $10 \%: 5 \mu \mathrm{g} / \mathrm{h}$ ). On a noté des améliorations par rapport aux valeurs de départ pour les indices douleur et invalidité (Pain Disability Index), douleur et sommeil (échelle analogique visuelle), l'Échelle d'incapacité du dos du Québec et le questionnaire SF-36 sur la santé, avec le STDB et avec le placebo, sans différence significative entre les traitements. Bien qu'on ait enregistré plus d'effets secondaires des opiacés avec le STDB qu'avec le placebo, aucune réaction indésirable grave n'est survenue. En tout, $82 \%$ des patients ont choisi de continuer le STDB dans le cadre d'une évaluation à long terme ouverte au cours de laquelle les améliorations sur les plans de l'intensité de la douleur, du statut fonctionnel et de la qualité de vie se sont maintenues pendant une période allant jusqu'à six mois, sans épuisement de l'effet analgésique.

CONCLUSION : Le STDB (de $5 \mu \mathrm{g} / \mathrm{h}$ à $20 \mu \mathrm{g} / \mathrm{h}$ ) représente une nouvelle option thérapeutique pour le traitement opiacé initial chez des patients souffrant de lombalgie chronique.

longer than three months. Frequently, however, low back pain does not fully resolve, and patients experience repeated exacerbations, with lifetime recurrences of up to $85 \%$ (2).

In chronic low back pain, treatment must be personalized and based on a combination of nonpharmacological and pharmacological approaches (3). The most frequently used ow back pain is a major health and socioeconomic problem, absenteeism and diminished quality of life. In Canada, four of five adults will experience at least one episode of back pain during their lives (1). The average sufferer usually has a long, episodic history. Chronic back pain is generally accepted as lasting
${ }^{1}$ Mount Sinai Hospital, Toronto, Ontario; ${ }^{2}$ University of Alberta Hospital, Edmonton, Alberta; ${ }^{3}$ London Health Sciences Centre, London,

Ontario; ${ }^{4}$ University of Calgary, Calgary, Alberta; ${ }^{5}$ Centre de Rhumatologie St-Louis, Ste Foy, Quebec; ${ }^{6}$ Purdue Pharma, Pickering, Ontario;

${ }^{7}$ Astellas Pharma US Inc, Deerfield, Illinois, USA

Correspondence: Dr John Eisenhoffer, Purdue Pharma, 575 Granite Court, Pickering, Ontario L1W 3W8. Telephone 1-800-387-5349,

fax 905-420-2503, e-mail john.eisenhoffer@purdue.ca 
categories of drug treatments are fixed nonopioid/opioid combinations (eg, codeine plus acetaminophen), nonopioid analgesics (ie, nonsteroidal anti-inflammatory drugs [NSAIDs]), and other drugs such as muscle relaxants, antidepressants and anticonvulsants (4).

NSAIDs, acetaminophen and muscle relaxants can be effective for the treatment of mild to moderate acute pain, but they are often suboptimal for the treatment of chronic, moderate to severe pain (5). The positive effects of muscle relaxants have been shown to be short lived (four to seven days), making them unsuitable for long-term use $(6,7)$. Longterm use of NSAIDs may lead to gastrointestinal complications, such as ulceration and bleeding $(8,9)$. In patients with a history of or increased risk for cardiovascular disease, the use of cyclooxygenase- 2 selective inhibitors are associated with an increased risk of cardiovascular events (10-12). A recent American Heart Association statement (13) recommended that NSAIDs be used for musculoskeletal pain only after failure of other treatments, and not as a first-line option.

Antidepressants, particularly those that act via noradrenergic mechanisms, are also used in the treatment of chronic low back pain (14-16), although a recent Cochrane review (17) concluded that there is no clear evidence to suggest that antidepressants are better than placebo as analgesics for low back pain. Furthermore, the occurrence of side effects, especially sedation and anticholinergic effects, limits their usefulness, particularly in the elderly.

The role of opioids in the treatment of chronic noncancer pain has been controversial. However, the American Pain Society recently published "Clinical Guidelines for the Use of Chronic Opioid Therapy in Chronic Noncancer Pain" (18) based on the recommendations of an expert panel, which concluded that opioids are safe and effective for carefully selected, well-monitored patients who have failed standard treatment. Recommendations for 'universal precautions' in the management of these patients are designed to balance the objectives of pain relief and improved functional outcomes with the risks of opioid therapy, including abuse and addiction (19). Immediaterelease formulations requiring multiple daily doses to maintain around-the-clock pain control are inconvenient, and decreased compliance may lead to misguided strategies to 'make up' missed doses or dosing that 'chases' pain symptoms. Long-acting opioids may provide advantages over short-acting opioids through a more sustained effect, greater convenience and improved adherence to the dosing regimen (19).

Buprenorphine is an opioid that recently (in 2007) became available in Canada for sublingual administration in patients with opioid dependency. This formulation is a combination of buprenorphine with naloxone and is designed to deter intravenous abuse. In Europe, sublingual and parenteral formulations have been available for pain management for approximately 20 years. Although buprenorphine has low oral bioavailability, its high lipid solubility makes it well suited for transdermal administration. A three-day patch formulation was introduced in Europe in 2003 for the treatment of chronic pain $(20,21)$. A greater understanding of the clinical role of buprenorphine in the treatment of chronic pain has developed (20-23). A recent European consensus statement (24) on pain management recommended buprenorphine, including transdermal formulations, as the first choice for opioid treatment in the elderly, noting that buprenorphine was the only opioid for which a dose adjustment was not required in patients with renal dysfunction.

Buprenorphine is a derivative of the alkaloid thebaine, and is a partial $\mathrm{mu}(\mu)$ - and ORL1-opioid receptor agonist, which also has kappa- and delta-opioid receptor antagonist activity $(20,25)$. Its high affinity for the receptors and slow dissociation from the $\mu$-receptor results in a relatively long duration of analgesic action. There have been concerns that this may also result in displacement of other opioids from the receptor and that in patients on full $\mu$-opioid agonists, the partial agonism of buprenorphine may then lead to withdrawal symptoms. Recent animal studies $(21,23,26)$ have not supported this drug interaction and suggest that $\mu$-opioid agonists and buprenorphine may, in fact, act synergistically.

Buprenorphine has also been considered to have an analgesic 'ceiling effect' (bell-shaped dose response curve). However, animal data (27) suggested that this may not be the case, and an analgesic 'ceiling effect' has not been demonstrated in humans within the therapeutic dose range $(21,22)$. However, a ceiling effect for respiratory depression has been observed with doses of buprenorphine well above the analgesic therapeutic dose (21). Clinical data suggest that respiratory depression may occur less frequently than with morphine, hydromorphone, methadone and transdermal fentanyl, although not when buprenorphine is combined with other central nervous system-depressant drugs (21). Previous reports that buprenorphine-induced respiratory depression is refractory to opioid antagonists may be a result of inadequate doses of the antagonist, given the unique receptor binding profile of buprenorphine. While a single bolus dose of naloxone will usually reverse respiratory depression associated with shortacting opioids, higher doses and continuous infusion are required with long-acting opioids and buprenorphine. If respiratory depression occurs with buprenorphine, it can be fully reversed with continuous infusion of high doses of naloxone (21).

A transdermal drug delivery system that provides three buprenorphine flux rates $(5 \mu \mathrm{g} / \mathrm{h}, 10 \mu \mathrm{g} / \mathrm{h}$ and $20 \mu \mathrm{g} / \mathrm{h}$; BuTrans, Purdue Pharma, Canada) has been developed and is designed to be worn for seven days. Pharmacokinetic studies have shown that stable plasma levels are maintained over this time period, thus avoiding the characteristic peaks and troughs produced by repeated sublingual or oral dosing. By avoiding hepatic firstpass metabolism, this formulation may avoid problems of oral dosing in patients with altered bioavailability due to comorbid gastrointestinal disease, and it may also have a particular advantage in patients who are vomiting or having difficulty swallowing. Previous studies with this seven-day buprenorphine transdermal system (BTDS) have demonstrated efficacy and tolerability in patients with osteoarthritis, compared with patients taking tramadol (28), and in a placebo-controlled maintenance of analgesia design, in patients with noncancer pain syndromes (29). The present study was designed as a placebo-controlled crossover study of analgesic efficacy and tolerability in patients with chronic low back pain.

\section{METHODS}

The objective of the present randomized, double-blinded, crossover study was to compare the efficacy and safety of BTDS 
versus placebo in patients with moderate to severe chronic low back pain. Patients who successfully completed the study were eligible for enrollment in a six-month open-label evaluation.

\section{Patients}

Men and nonpregnant women older than 18 years of age with low back pain of at least moderate severity ( 2 on a five-point ordinal scale) for more than six weeks duration that was inadequately treated with nonopioids were enrolled. Baseline assessments included an evaluation of whether the pain represented a neuropathic or nociceptive etiology. Patients whose analgesic requirement was expected to exceed the maximum BTDS dose, or who were refractory to opioid therapy or had an allergy to acetaminophen or opioids were excluded from the study. Patients who were undergoing any procedures or treatments (such as physiotherapy or surgery) that were likely to affect their pain, or those with a significant alternative source of pain, were also excluded, as were patients who would require the use of external heat sources. Other exclusion criteria included elevated liver function tests, severe organ dysfunction, head injury or seizures, chronic obstructive pulmonary disease, asthma, respiratory depression, cor pulmonale, heart failure, peptic ulcer disease or gastrointestinal tract inflammation. Patients with suspected psychological dependence on narcotic drugs or alcohol, or with a history of major psychiatric disorders were also excluded. Research ethics boards at the six participating centres approved the protocol and informed consent. Each patient gave written informed consent before participating in the present study.

\section{Medications}

Medications included active BTDS in $5 \mu \mathrm{g} / \mathrm{h}, 10 \mu \mathrm{g} / \mathrm{h}$ and $20 \mu \mathrm{g} / \mathrm{h}$ patches (BuTrans 5, 10 and 20 [marketed as Norspan in some regions outside of North America], Purdue Pharma, Canada) and matching placebo patches. All patients were randomly assigned to receive either active or placebo BTDS at an initial dose of $5 \mu \mathrm{g} / \mathrm{h}$, and all patches were to be worn for a period of seven days. The dose was titrated weekly to the maximum tolerated dose $(5 \mu \mathrm{g} / \mathrm{h}, 10 \mu \mathrm{g} / \mathrm{h}$ or $20 \mu \mathrm{g} / \mathrm{h})$ based on pain relief and side effects. For any breakthrough pain, patients were provided with codeine plus acetaminophen tablets (30 mg/300 mg, Tylenol No 3, McNeil Consumer Healthcare, Canada) for rescue analgesia (one to two tablets every $4 \mathrm{~h}$ to $6 \mathrm{~h}$ as required). At the end of the double-blinded treatment period, patients who chose to receive long-term open-label BTDS were initiated at a dose of $5 \mu \mathrm{g} / \mathrm{h}$, which was titrated every one to two weeks to a maximum of $40 \mu \mathrm{g} / \mathrm{h}$.

\section{Study design}

The present study was a randomized, double-blinded, crossover comparison of the efficacy and safety of seven-day BTDS and placebo. Patients were withdrawn from all prestudy opioids the evening before random assignment to receive either active or placebo BTDS at a starting dose of $5 \mu \mathrm{g} / \mathrm{h}$. Nonopioid analgesics that had been administered at a stable dose for two weeks before enrollment were permitted at that stable dose throughout the study. Patients returned to the clinic for weekly visits, and after four weeks, they were switched over to the alternate treatment for another four weeks, following which, they were eligible to participate in a six-month open-label evaluation. A blocked randomization procedure was used to generate the treatment allocation listing. For every four successive patients, two received active BTDS in the first phase and two received active BTDS in the second phase. There were 100 patient numbers generated. The generation of the random code was facilitated by the use of PROC PLAN in SAS version 6.12 (SAS Institute Inc, USA).

Patients recorded their pain intensity in a diary twice a day (08:00 and 20:00) using a five-point ordinal scale $(0=$ none, $1=$ mild, $2=$ moderate, $3=$ severe and $4=$ excruciating) and a visual analogue scale (VAS). The VAS was a $100 \mathrm{~mm}$ unmarked line, bounded on the left by 'no pain' and on the right by 'excruciating pain'. Nausea was also assessed at 08:00 and 20:00 in the diary with a $100 \mathrm{~mm}$ VAS (anchors 'no nausea' and 'severe nausea'). Drowsiness was assessed at 20:00 (100 mm VAS; anchors 'no drowsiness' and 'extreme drowsiness'), and patients were asked how well rested they felt at 08:00 daily (100 mm VAS; anchors 'very well rested' and 'not at all well rested'). Pain intensity was also assessed at the weekly clinic visits during the double-blinded phase and at bimonthly visits during open-label treatment, using a ordinal scale and a VAS scale as described above. All other assessments were completed at baseline, crossover and end of study visits in the double-blinded phase and at two, four and six months during open-label treatment.

Patients assessed their pain-related disability using the Pain Disability Index (PDI) $(30,31)$, which consists of seven disability subscales, each representing a different area of everyday functioning: family/home responsibilities, recreation, social activity, occupation, sexual behaviour, self-care and life support activity. Each scale was graded from 0 to 10, in which 0 indicated no disability and 10 indicated total disability. The seven subscales were summed to yield an overall disability score (0 to 70). Functional ability was also assessed using the Quebec Back Pain Disability Scale (32), which consists of 20 items rated on a six-point ordinal scale $(0=$ no difficulty, $1=$ minimally difficult, 2 = somewhat difficult, 3 = fairly difficult, $4=$ very difficult and $5=$ unable to do).

The impact of pain on sleep was assessed with a $100 \mathrm{~mm}$ VAS (anchors 'never' to 'always') for each of the following areas: 'Trouble falling asleep due to pain', 'Needed pain medication to sleep', 'Needed sleeping medication to sleep', 'Awakened by pain in night', 'Awakened by pain in the morning' and 'How often was partner sleep disturbed'. The duration of sleep was assessed by asking patients, 'On average, how many hours of sleep are you getting each night?'.

Patients' level of activity was assessed with a $100 \mathrm{~mm}$ VAS (anchors 'inactive/sedentary' to 'very active/daily vigorous activity') and they were also asked about their employment status.

Health status was assessed using the Short-Form 36 Health Survey (SF-36) (33), a general health status outcome measure, which includes one multi-item scale measuring each of eight concepts: physical functioning, role limitations due to physical health problems, bodily pain, general health, vitality (energy/fatigue), social functioning, role limitations due to emotional problems, and mental health (psychological distress and well-being).

Effectiveness of treatment was assessed by both the patient and investigator using a four-point ordinal scale $(0=$ not effective, 1 = slightly effective, 2 = moderately effective and 


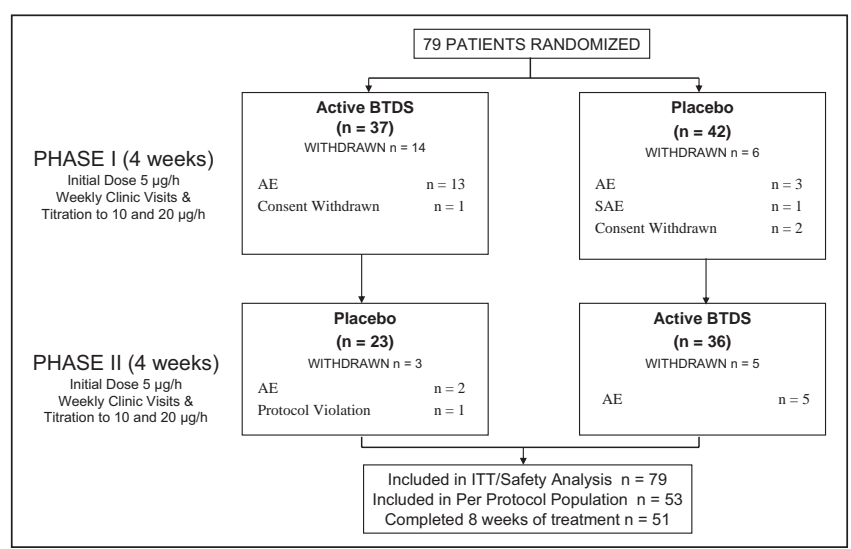

Figure 1) Study design and patient disposition. AE Adverse events; BTDS Buprenorphine transdermal system; ITT Intent-to-treat; SAE Severe adverse events

3 = highly effective). Overall treatment preference was assessed at the end of the study by both patients and investigators (phase 1, phase 2 or no preference) without unblinding the treatment assignment.

The occurrence and severity of any adverse events were assessed at all clinic visits using a nondirected questionnaire.

\section{Data analysis}

The primary measures of efficacy were the VAS and five-point ordinal pain intensity scales from the patient daily diaries averaged over the last week of treatment in each phase. It was estimated that with 40 patients completing the study, there would be $80 \%$ power $(\beta=0.20)$ at the $5 \%$ significance level $(\alpha=0.05)$ to detect a difference of $15 \mathrm{~mm}$ in VAS pain intensity.

Demographic characteristics of patients were summarized using descriptive statistics. The pain intensity (VAS and ordinal), nausea (VAS) and drowsiness (VAS) scores from the patient's diaries were averaged by week and time (08:00 and 20:00), and overall. Because the dose of buprenorphine was titrated during each phase, the efficacy data from the last week of treatment in each phase served as the primary basis for comparison between the groups. For patients who withdrew from the study, the final week of treatment was considered to be the last week, for analysis purposes, provided that they had received a minimum of two weeks in the second phase. The per protocol analysis was performed for patients with no major protocol violations who completed at least two consecutive weeks of treatment in each phase of the study using data from their last week of treatment. An intent-to-treat (ITT) analysis that included all patients who received at least one dose of study medication was conducted for efficacy and safety. Additional subgroup analyses were performed for patients who completed a full four weeks in each phase and for comparison of pain intensity and BTDS dose in opioid-tolerant and opioid-naive patients. For open-label data analysis, all patients who completed at least one visit were included in the efficacy analysis, and all patients who received at least one dose of study medication were included in the safety analysis.

The diary scores were averaged across the two assessment times on each day, and the daily scores were averaged to derive weekly scores. Three-way ANOVA was used to test for the effect of treatment, phase and sequence (carryover) using data from the mean scores by treatment. The patient withinsequence variance was used as the error term for testing carryover effect. Multivariate repeated measures analysis was used to test for the effects of drug, week, time of day and their interactions. The use of rescue (number of tablets and frequency of use) was compared by treatment group based on average daily consumption. ANOVA and multivariate repeated measures ANOVA were used to compare rescue use averaged over treatment and by week. Treatment phase preference rates were compared using the binomial test.

The scores from all clinic assessments, including pain intensity (VAS and ordinal scale), PDI, Quebec Back Pain Disability Scale, Pain and Sleep, level of activity, SF-36 and effectiveness ratings, were analyzed using three-way ANOVA as described above. The mean ratings at the end of the double-blinded period were used as the baseline for comparison with the mean ratings at each visit in the open-label phase and the average across all open-label visits, using the paired $t$ test.

Adverse events were listed by patient and by order of frequency, as defined by the number of patients experiencing adverse events, the number of events reported and the severity. All adverse events were coded using preferred terms based on Coding Symbols for a Thesaurus of Adverse Reaction Terms, Fourth Edition to standardize the terminology. McNemar's $\chi^{2}$, which requires that patients be exposed to both treatments, was used to determine the significance of differences in overall frequency of side effects between the two treatment groups. Statistical significance was defined as $\mathrm{P}<0.05$ for a two-tailed hypothesis.

\section{RESULTS}

Seventy-nine patients enrolled in the study; 46 (58\%) were opioid naive, and 33 (42\%) were already receiving opioid therapy before enrollment in the current study. Thirty-eight per cent of enrolled patients began the study with severe or excruciating pain. Of these randomly assigned patients, 53 were evaluable for efficacy and safety in the per protocol population (28 men, 25 women, mean $[ \pm \mathrm{SD}]$ age $54.5 \pm 12.7$ years), having completed at least two consecutive weeks in each phase. There were 51 patients who completed four weeks of treatment in each phase. In the per protocol population, the average duration of low back pain was $14.1 \pm 10.7$ years (minimum 1.4 years; maximum 53.1 years). Twenty-two patients (42\%) were receiving opioids at the time of enrollment into the study, and 31 (58\%) were opioid naive. Thirteen patients were assessed as having neuropathic pain for an average of $17.9 \pm 26.5$ years, and 50 patients were assessed as having nociceptive pain for an average of $17.7 \pm 23.0$ years (some patients had both neuropathic and nociceptive pain; therefore, the total number exceeds the actual number of patients in the per protocol population). Twenty-eight patients withdrew after random assignment -24 due to adverse events (18 from active BTDS and six from placebo), three withdrew consent and one was withdrawn for a protocol violation. All 79 patients were evaluable for efficacy and safety in the ITT population. Patient disposition is presented in Figure 1.

At baseline, 23 patients (43.4\%) were employed, 13 patients (24.5\%) were retired and 17 patients $(32.1 \%)$ were unemployed. Of the unemployed patients, 13 of 17 patients (76.5\%) reported that their unemployment was related to their pain. 


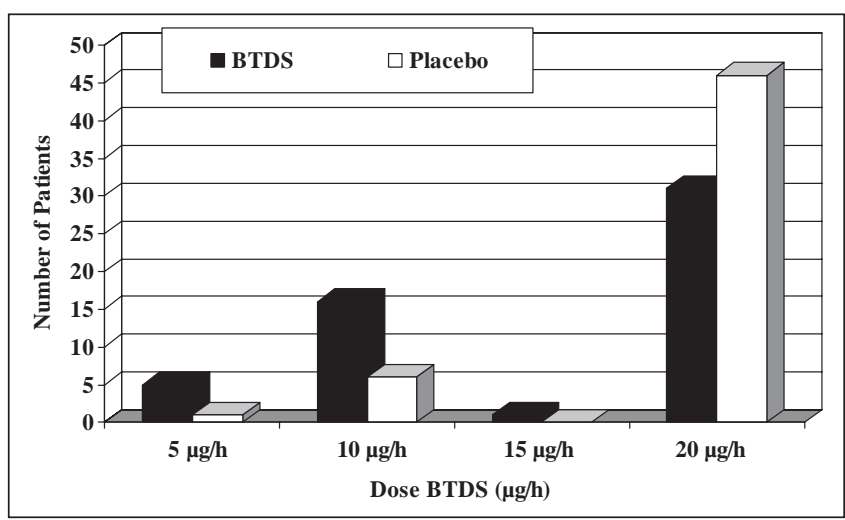

Figure 2) Distribution of final doses for the per protocol population. BTDS Buprenorphine transdermal system

TABLE 1

Mean weekly pain intensity score recorded in a daily diary

\begin{tabular}{lcccc}
\hline & Baseline & BTDS & Placebo & $\mathbf{P}^{*}$ \\
\hline \multicolumn{1}{l}{ Per protocol - last week } & & & \\
\hline Ordinal & $2.5 \pm 0.6$ & $1.7 \pm 0.6$ & $2.0 \pm 0.7$ & 0.0358 \\
VAS, mm & $62.1 \pm 15.5$ & $37.6 \pm 20.7$ & $43.6 \pm 21.2$ & 0.0487 \\
ITT - last week & & & & \\
\hline Ordinal & $2.5 \pm 0.6$ & $1.8 \pm 0.6$ & $2.0 \pm 0.7$ & 0.0226 \\
VAS, mm & $61.4 \pm 16.4$ & $40.2 \pm 20.2$ & $44.4 \pm 20.2$ & 0.0919 \\
Per protocol - week 4 & & & & \\
\hline Ordinal & $2.5 \pm 0.6$ & $1.8 \pm 0.6$ & $2.0 \pm 0.7$ & 0.0180 \\
VAS, mm & $62.1 \pm 15.5$ & $38.0 \pm 20.5$ & $43.6 \pm 21.4$ & 0.0121 \\
ITT - week 4 & & & & \\
\hline Ordinal & $2.5 \pm 0.6$ & $1.8 \pm 0.6$ & $2.0 \pm 0.7$ & 0.0188 \\
VAS, mm & $61.4 \pm 16.4$ & $39.2 \pm 20.5$ & $43.9 \pm 21.3$ & 0.0124 \\
\hline
\end{tabular}

*Between treatments. BTDS Buprenorphine transdermal system; ITT Intent-totreat; VAS Visual analogue scale

Patients had been unemployed for an average of $5.7 \pm 7.2$ years, and there was no change in the patients' employment status over the course of the crossover study.

The mean daily transdermal buprenorphine dose for the last week of each treatment phase was significantly lower in the BTDS group than in the placebo group for both the per protocol population $(15.5 \pm 5.7 \mu \mathrm{g} / \mathrm{h}$ versus $18.6 \pm 3.7 \mu \mathrm{g} / \mathrm{h}, \mathrm{P}=0.0018)$ and the ITT population $(14.3 \pm 6.3 \mu \mathrm{g} / \mathrm{h}$ versus $16.8 \pm 5.5 \mu \mathrm{g} / \mathrm{h}$, $\mathrm{P}=0.0174$ ). There was no evidence of a carryover effect in either population (per protocol, $\mathrm{P}=0.5458$; ITT, $\mathrm{P}=0.8120$ ). As shown in Figure 2, the majority of patients in both populations used the $20 \mu \mathrm{g} / \mathrm{h}$ BTDS patch, but the proportion was higher in the placebo group ( $87 \%$ versus 59\%). The mean BTDS doses administered to the opioid-naive patients $(58 \%)$ and opioidtolerant patients $(42 \%)$ were comparable $(14.0 \pm 6.1 \mu \mathrm{g} / \mathrm{h}$ versus $14.8 \pm 6.6 \mu \mathrm{g} / \mathrm{h})$.

There was a trend toward greater use of rescue $30 \mathrm{mg}$ codeine plus $300 \mathrm{mg}$ acetaminophen tablets in the placebo group than in the active BTDS group during the last week of treatment, but this did not reach statistical significance. Patients in the per protocol population used $1.8 \pm 2.6$ tablets/day of $30 \mathrm{mg}$ codeine plus $300 \mathrm{mg}$ acetaminophen in the BTDS phase, and $2.4 \pm 2.8$ tablets/day in the placebo phase $(\mathrm{P}=0.0717)$.

Baseline pain scores for the per protocol population were $62.1 \pm 15.5 \mathrm{~mm}$ on the VAS and $2.5 \pm 0.6$ on the ordinal scale. During the last week of treatment, the mean VAS pain
TABLE 2

Mean weekly pain intensity score recorded at clinic visits

\begin{tabular}{lcccc}
\hline & Baseline & BTDS & Placebo & $\mathbf{P}^{*}$ \\
\hline Per protocol - last week & & & & \\
\hline Ordinal & $2.5 \pm 0.6$ & $1.7 \pm 0.7$ & $1.9 \pm 0.7$ & 0.0605 \\
VAS, mm & $62.1 \pm 15.5$ & $39.9 \pm 19.9$ & $46.9 \pm 22.4$ & 0.0534 \\
ITT - last week & & & & \\
\hline Ordinal & $2.5 \pm 0.6$ & $1.8 \pm 0.7$ & $2.1 \pm 0.7$ & 0.0290 \\
VAS, mm & $61.4 \pm 16.4$ & $42.3 \pm 20.1$ & $48.4 \pm 21.7$ & 0.0241 \\
Per protocol - week 4 & & & & \\
\hline Ordinal & $2.5 \pm 0.6$ & $1.7 \pm 0.7$ & $2.0 \pm 0.7$ & 0.0285 \\
VAS, mm & $62.1 \pm 15.5$ & $40.5 \pm 20.3$ & $47.5 \pm 22.7$ & 0.0197 \\
ITT - week 4 & & & & \\
\hline Ordinal & $2.5 \pm 0.6$ & $1.8 \pm 0.7$ & $2.0 \pm 0.7$ & 0.0553 \\
VAS, mm & $61.4 \pm 16.4$ & $40.9 \pm 20.2$ & $48.0 \pm 22.5$ & 0.0269 \\
\hline
\end{tabular}

${ }^{*}$ Between treatments. BTDS Buprenorphine transdermal system; ITT Intentto-treat; VAS Visual analogue scale

intensity scores recorded in the daily diaries were lower in the active BTDS group than in the placebo group $(37.6 \pm 20.7 \mathrm{~mm}$ versus $43.6 \pm 21.2 \mathrm{~mm}, \mathrm{P}=0.0487$ ) (Table 1 ). The corresponding decreases from baseline were $39.5 \%$ and $29.8 \%$, respectively $(\mathrm{P}=0.0001$ in each case). There was no evidence of a carryover effect $(\mathrm{P}=0.8347)$. Similarly, during the last week of treatment, mean ordinal pain intensity scores from the daily diaries were lower in the active BTDS phase than the placebo phase $(1.7 \pm 0.6$ versus $2.0 \pm 0.7, \mathrm{P}=0.0358)$ (Table 1$)$, with decreases from baseline of $32.0 \%$ and $20.0 \%$, respectively $(\mathrm{P}=0.0001$ in each case). There was no evidence of a carryover effect $(\mathrm{P}=0.8508)$. Similar statistically significant reductions in pain scores on BTDS compared with placebo were also observed in week 4 , as shown in Table 1.

For patients who titrated up to the maximum dose of $20 \mu \mathrm{g} / \mathrm{h}$, the change in daily VAS pain intensity score from baseline was greater as the transdermal dose increased while receiving active BTDS compared with placebo. The reduction in pain intensity scores from baseline was $17.2 \mathrm{~mm}$ and $21.8 \mathrm{~mm}$ for the $5 \mu \mathrm{g} / \mathrm{h}$ and $20 \mu \mathrm{g} / \mathrm{h}$ doses, respectively, in the active BTDS group, as opposed to $14.7 \mathrm{~mm}$ and $15.6 \mathrm{~mm}$ for the corresponding doses in the placebo group.

In the BTDS treatment group, pain scores did not change by day across the seven-day treatment period. The mean change from baseline in VAS daily pain scores for the first four days of treatment was $11.7 \mathrm{~mm}$ compared with $11.4 \mathrm{~mm}$ during the last three days. Similar results were shown for mean change from baseline in daily ordinal pain scores $(0.55$ versus 0.53).

In patients who were receiving opioids at the time of study entry, the mean change from baseline in VAS score during BTDS treatment was $23.1 \pm 20.2 \mathrm{~mm}(34.6 \%)$, which was similar to scores in patients who were opioid naive $(25.4 \pm 22.4 \mathrm{~mm})$ (43.0\%).

Pain intensity was also assessed at the weekly clinic visits and showed similar improvements with BTDS compared with placebo. The mean VAS and ordinal pain scores over the previous week measured during the last week of treatment and in week 4 of treatment are shown in Table 2, for the per protocol and ITT populations.

While there were no differences in any of the seven subscales or in the total score of the PDI during active BTDS treatment compared with placebo $(\mathrm{P}=0.4860)$, there was 


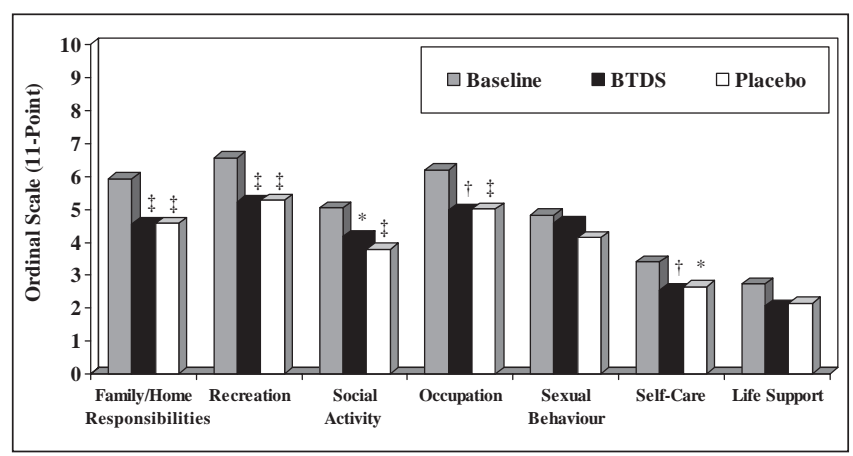

Figure 3) Pain Disability Index scores at the last week of treatment (per protocol population). $* P<0.05 ; \dagger P<0.005 ;{ }^{\star} \mathrm{P}<0.0005$ change from baseline. BTDS Buprenorphine transdermal system

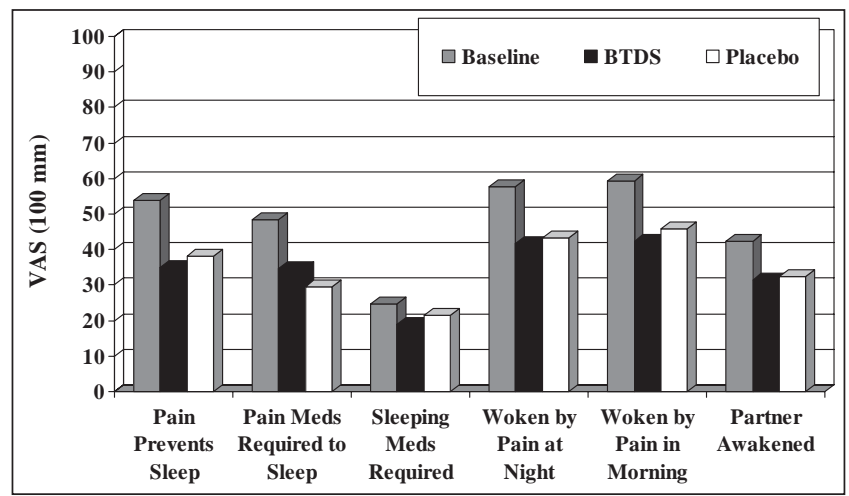

Figure 4) Pain and Sleep questionnaire scores at the last week of treatment (per protocol population). BTDS Buprenorphine transdermal system; Meds Medications; VAS Visual analogue scale

a significant change from baseline in both groups measured during the last week of treatment (Figure 3). The percentage change from baseline for the overall PDI score was $19.6 \%$ for active BTDS $(\mathrm{P}=0.0002)$ and $21.0 \%$ for placebo $(\mathrm{P}=0.0001)$.

The baseline score for the Quebec Back Pain Disability Scale was $2.7 \pm 0.9$. In the last week of treatment, scores were $2.3 \pm 0.9$ for the BTDS group and $2.4 \pm 1.0$ for the placebo group in the per protocol population. Scores improved by $12.8 \%$ in the active BTDS treatment phase $(\mathrm{P}=0.0014)$ and by $8.2 \%$ in the placebo phase $(\mathrm{P}=0.0055)$ compared with baseline scores, but there was no difference between the treatments $(\mathrm{P}=0.2995)$.

There were improvements from baseline in all six items of the Pain and Sleep questionnaire following active BTDS or placebo treatment (Figure 4), but there were no differences between treatment groups. The overall Pain and Sleep summary scores decreased from baseline $(244.5 \pm 126.3 \mathrm{~mm})$ by $29.5 \%$ for active BTDS $(172.4 \pm 122.8 \mathrm{~mm}, \mathrm{P}=0.0001)$ and $27.1 \%$ for placebo $(178.2 \pm 112.6 \mathrm{~mm}, \mathrm{P}=0.0001)$. Patients were asked in their daily diaries how well rested they felt at 08:00 each day. There was a trend toward patients feeling more rested after active BTDS treatment $(35.1 \pm 25.2 \mathrm{~mm})$ than after placebo $(39.0 \pm 27.7 \mathrm{~mm})$, as measured during week 4 of treatment $(\mathrm{P}=0.0720)$ in the per protocol population.

The SF-36 was administered at baseline, crossover and the end of the study. In all eight domains, scores were better following either BTDS or placebo than at baseline (Figure 5). There were no significant differences between active BTDS and

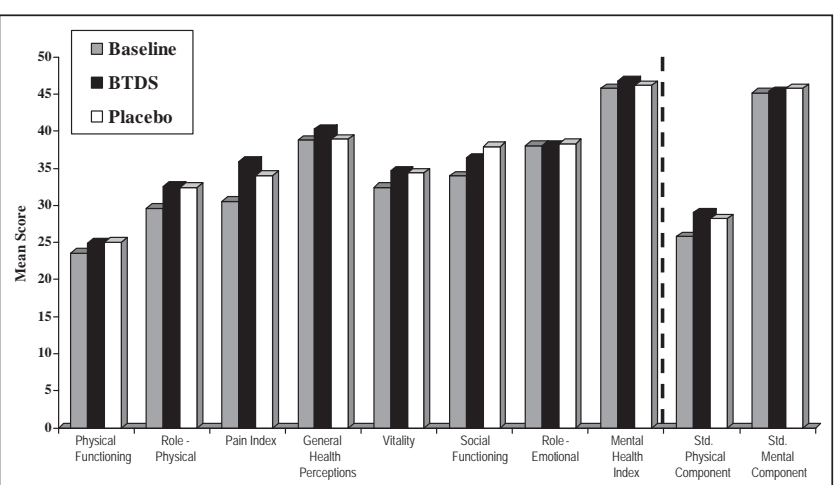

Figure 5) Short-Form 36 Health Survey scores at the last week of treatment (per protocol population). Higher scores indicate a better health state. Data normalized to Canadian standard (std). BTDS Buprenorphine transdermal system

placebo treatments for all of the domains normalized to Canadian standards for the per protocol population during the last week of treatment. There was no evidence of a carryover effect for any of the subscales $(\mathrm{P}>0.1691)$.

The baseline activity level for the per protocol population was $39.9 \pm 23.8 \mathrm{~mm}$. The patients' activity levels increased by $9.8 \%$ for active BTDS $(43.8 \pm 23.0 \mathrm{~mm})$ and by $10.1 \%$ for placebo $(43.9 \pm 23.7 \mathrm{~mm})$ compared with baseline. There were no significant differences between the treatment groups $(\mathrm{P}=0.9355)$ and no carryover effect $(\mathrm{P}=0.5061)$.

Both patients and investigators rated the clinical effectiveness of their current treatment at baseline and at the end of each phase. At baseline, the mean effectiveness rating of the patients' prestudy analgesic was $1.2 \pm 0.8$ for the patients and $1.1 \pm 0.8$ for the investigators. At the end of each phase, the mean scores for patient ratings were $1.3 \pm 1.1$ and $0.9 \pm 1.0$ for active BTDS and placebo, respectively $(\mathrm{P}=0.1782)$. The mean scores for investigator ratings were $1.2 \pm 1.0$ and $0.9 \pm 1.0$ for active BTDS and placebo, respectively $(\mathrm{P}=0.1221)$. Forty-five per cent of patients receiving BTDS and 30\% of patients receiving placebo rated their treatment as moderately or highly effective. For investigators, 43\% rated BTDS and 30\% rated placebo as moderately or highly effective.

Forty-three per cent of the per protocol patients preferred the phase in which they received BTDS, 38\% preferred the placebo phase and $19 \%$ had no preference $(\mathrm{P}=0.6473)$. Similarly, investigators preferred the phase in which patients were given BTDS for $43 \%$ of patients, placebo for $36 \%$ of patients and had no preference in $21 \%$ of cases $(\mathrm{P}=0.5371)$.

There was no difference in the overall mean nausea scores during the last week of treatment for the per protocol population $(8.5 \pm 11.1 \mathrm{~mm}$ versus $6.1 \pm 9.2 \mathrm{~mm}$ for BTDS and placebo, respectively, $\mathrm{P}=0.2962)$. Patients felt drowsier with active BTDS $(24.3 \pm 19.5 \mathrm{~mm})$ than with placebo $(16.9 \pm 18.4 \mathrm{~mm})$ during the last week of treatment in each phase for the per protocol population $(\mathrm{P}=0.0066)$.

There were 406 adverse events reported by 72 patients during active BTDS treatment, and 318 events reported by 58 patients during placebo treatment. The number of patients reporting at least one event was significantly greater during BTDS treatment $(\mathrm{P}=0.0143)$. Thirty-eight $(53 \%)$ patients from the BTDS group and 24 (42\%) patients from the placebo group 
TABLE 3

Incidence of most common adverse events*

\begin{tabular}{|c|c|c|c|c|c|}
\hline & BTDS & $\begin{array}{c}\text { Mean } \\
\text { maximum } \\
\text { severity }\end{array}$ & Placebo & $\begin{array}{c}\text { Mean } \\
\text { maximum } \\
\text { severity }\end{array}$ & $\mathbf{P}$ \\
\hline Nausea & $28(38.4)$ & 2.0 & 11 (16.9) & 1.0 & 0.0330 \\
\hline Somnolence & $22(30.1)$ & 2.3 & $4(6.2)$ & 1.9 & 0.0010 \\
\hline Pruritus & $22(30.1)$ & 1.3 & $18(27.7)$ & 1.7 & 0.8084 \\
\hline Asthenia & $21(28.8)$ & 2.0 & $10(15.4)$ & 2.3 & 0.1336 \\
\hline Constipation & $20(27.4)$ & 1.6 & $14(21.5)$ & 1.6 & 0.2207 \\
\hline Insomnia & $18(24.7)$ & 2.3 & 17 (26.2) & 1.9 & 1.000 \\
\hline Dizziness & $16(21.9)$ & 2.5 & $5(7.7)$ & 1.4 & 0.1797 \\
\hline Sweating & $15(20.6)$ & 2.3 & $10(15.4)$ & 1.7 & 0.2253 \\
\hline Pain & $14(19.2)$ & 2.4 & $18(27.7)$ & 2.3 & 0.2253 \\
\hline Anorexia & $14(19.2)$ & 2.0 & $12(18.5)$ & 1.5 & 0.5930 \\
\hline Vomiting & $11(15.1)$ & 2.0 & $3(4.6)$ & 2.7 & 0.0956 \\
\hline Yawn & $11(15.1)$ & 1.3 & $12(18.5)$ & 1.4 & 1.000 \\
\hline Nervousness & $10(13.7)$ & 1.8 & $14(21.5)$ & 1.8 & 0.2850 \\
\hline Rash & $9(12.3)$ & 1.6 & $7(10.8)$ & 1.4 & 0.7389 \\
\hline Headache & $8(11.0)$ & 2.3 & $6(9.2)$ & 1.8 & 1.000 \\
\hline Diarrhea & $8(11.0)$ & 2.1 & $13(20.0)$ & 1.4 & 0.2482 \\
\hline Overall & 72 (98.6) & 2.3 & $58(89.2)$ & 2.2 & 0.0143 \\
\hline
\end{tabular}

Data presented as $n$ (\%) unless otherwise indicated. ${ }^{*}$ Based on 73 patients who received buprenorphine transdermal system (BTDS) and 65 patients who received placebo

described their adverse events as severe. The most frequent adverse events are listed in Table 3.

Skin reactions at the current or previous patch sites were experienced by $24(30.3 \%)$ and 19 (24.1\%) patients during the active BTDS and placebo phases, respectively. Most reactions occurred at the current application site (22/24 for BTDS and $18 / 19$ for placebo), and were not severe, except for one event that occurred during BTDS and four during placebo treatment. The following skin reactions were reported during active BTDS and placebo phases, respectively: pruritus $(n=19, n=16)$, rash $(n=8, n=6)$, pain $(n=4, n=2)$, ecchymosis $(n=2, n=0)$, vesiculobullous rash $(n=1, n=1)$, maculopapular rash $(n=0$, $\mathrm{n}=1)$, skin discolouration $(\mathrm{n}=1, \mathrm{n}=0)$ and dry skin $(\mathrm{n}=1$, $\mathrm{n}=0$ ).

One serious adverse event was reported during the course of the trial, while a patient was receiving $5 \mu \mathrm{g} / \mathrm{h}$ placebo BTDS patch. The patient experienced severe shortness of breath and urinary retention after three days of wearing the BTDS patch, and the patch was removed five days later at the patient's regularly scheduled visit, at which time the patient was withdrawn from the study. No treatment was given for the events, and the patient recovered completely one day after the placebo BTDS patch was removed. There were no serious adverse events reported during active BTDS treatment.

Of the 51 patients who completed the entire eight-week study period, $42(82 \%)$ chose to continue to receive BTDS in the long-term open-label extension of the study and were treated for a mean of $132.4 \pm 57.7$ days. Twenty-one patients completed six months of open-label treatment (mean $179.1 \pm 11.6$ days) at a final dose of $14.5 \pm 6.1 \mu \mathrm{g} / \mathrm{h}$, compared with a mean final dose in the double-blinded phase of $15.2 \pm 6.0 \mu \mathrm{g} / \mathrm{h}$ and a final dose of $14.3 \pm 5.7 \mu \mathrm{g} / \mathrm{h}$ for all evaluable patients who received open-label treatment.

The mean VAS pain intensity scores over the previous $24 \mathrm{~h}$ were significantly lower at all open-label visits (two, four and
TABLE 4

Clinical benefit of buprenorphine transdermal system throughout long-term open-label treatment

\begin{tabular}{lccc}
\hline & $\begin{array}{c}\text { End of } \\
\text { double- } \\
\text { blinded trial }\end{array}$ & $\begin{array}{c}\text { Last open- } \\
\text { label trial } \\
\text { visit }\end{array}$ & P \\
\hline VAS pain intensity (past week) & $38.5 \pm 17.9$ & $32.9 \pm 28.1$ & 0.5431 \\
Ordinal pain intensity (past week) & $1.6 \pm 0.6$ & $1.6 \pm 0.8$ & 0.8454 \\
VAS pain intensity (past 24 h) & $39.4 \pm 19.1$ & $13.2 \pm 20.2$ & 0.0001 \\
Ordinal pain intensity (past 24 h) & $1.7 \pm 0.6$ & $1.6 \pm 0.9$ & 0.5253 \\
Overall Pain and Sleep & $156.0 \pm 113.7$ & $146.3 \pm 116.8$ & 0.5647 \\
Overall pain and disability & $26.9 \pm 15.2$ & $25.6 \pm 18.3$ & 0.5767 \\
Average Quebec Back Pain Disability & $2.2 \pm 0.9$ & $2.2 \pm 1.1$ & 0.9150 \\
$\quad$ Scale & & & \\
SF-36 standard physical component & $28.6 \pm 9.3$ & $25.3 \pm 10.7$ & 0.0226 \\
SF-36 standard mental component & $47.6 \pm 12.8$ & $49.3 \pm 17.9$ & 0.9734 \\
\hline
\end{tabular}

Data presented as mean \pm SD. SF-36 Short-Form 36 Health Survey; VAS Visual analogue scale

six months) and at the last visit, than during the double-blinded phase $(\mathrm{P}=0.0001)$. There were no other differences in pain intensity or other functional assessments between the openlabel treatment phase and baseline (end of the double-blinded phase), with the exception of the SF-36 standardized physical component, which was significantly lower in the last week of open-label treatment than at baseline (Table 4). At the end of the open-label treatment phase, 34 patients $(82 \%)$ reported receiving a great or moderate benefit from BTDS.

A total of 21 adverse events were reported by 40 patients during open-label treatment, with a mean maximum severity of 2.4. The most common events were application site reactions. Rash was reported most frequently (42.9\% of patients), followed by pruritus $(33.3 \%)$. Systemic side effects included constipation (28.6\%), nausea (23.8\%), asthenia (10.0\%), dizziness $(14.3 \%)$, and insomnia, pain and somnolence (11.0\% each).

\section{DISCUSSION}

The present randomized, double-blinded, crossover study demonstrates the efficacy and safety of BTDS in the treatment of moderate to severe chronic low back pain. The lower pain intensity scores with active BTDS treatment versus placebo were statistically different, although both active and placebo groups were given codeine plus acetaminophen, as required, for rescue analgesia. Both treatment groups experienced statistically significant improvements from baseline with respect to quality of life and functional disability measures; however, there were no differences between treatment groups.

There has been much discussion in the literature on what constitutes a clinically meaningful change in pain scores. Recommendations have ranged from a reduction of $30 \%$ to $50 \%$ in the setting of a clinical trial (34-36). In keeping with these bounds, active BTDS produced a $39.5 \%$ change from baseline for VAS scores and a $32.0 \%$ change from baseline for ordinal scores, indicating that the response to active BTDS was both statistically and clinically meaningful.

While there was a significant placebo response in the present study (29.8\% change from baseline on VAS scale), the measurement of response, in both active and placebo groups, is complicated by the consumption of active rescue medication (300 $\mathrm{mg}$ acetaminophen plus $30 \mathrm{mg}$ codeine). Due to the ethical concerns of placebo-controlled studies, an active opioid 
rescue was used. In the present study, the higher consumption of active rescue medication in the placebo phase did not reach statistical significance. Recent clinical data have suggested that coadministration of a full $\mu$-receptor agonist and buprenorphine (a partial $\mu$-receptor agonist) does not interfere with the analgesic effects of either opioid $(21,23,26)$. Therefore, although the use of acetaminophen plus codeine in the BTDS phase of the present study is not considered to have attenuated the effect of either opioid, the greater use of opioid rescue medication in the placebo group is considered to have potentially diminished the difference in analgesic response between the two treatment groups. For example, in a study comparing controlled-release oxycodone (OxyContin, Purdue Pharma) with placebo in painful diabetic neuropathy, plain acetaminophen was used as a rescue analgesic, and a difference of $30 \mathrm{~mm}$ in VAS pain intensity was detected between treatments (37). In contrast, in a study comparing controlled-release oxycodone with placebo in low back pain, in which active codeine plus acetaminophen and active immediate-release oxycodone were used as rescue in the placebo and active arms of the study, respectively, the difference in VAS pain intensity between the two treatments was $11 \mathrm{~mm}$ (38). Although the different pain populations may have contributed to this difference, it is likely that the use of an active opioid rescue in the study of low back pain could have masked, to some degree, the true difference between the test analgesic and placebo. Nevertheless, in the present study, the primary outcome measures were significantly better with active BTDS than with placebo, even with the use of active codeine plus acetaminophen as a rescue analgesic.

When considering the difference in average pain scores between treatment groups, it is equally important to note that the change from baseline while on BTDS is a function of the distribution of pain scores at baseline. Patients with higher baseline values elicit numerically greater changes from baseline. A subgroup analysis from the per protocol population showed that BTDS elicited a $18.6 \mathrm{~mm}$ change from baseline in patients with moderate baseline pain (25/53 patients; baseline VAS = $53.0 \mathrm{~mm}$ ), a $32.4 \mathrm{~mm}$ change from baseline in patients with severe baseline pain (24/53 patients; baseline VAS $=71.2 \mathrm{~mm}$ ) and a $23.9 \mathrm{~mm}$ change from baseline in patients with excruciating baseline pain ( $3 / 53$ patients; baseline VAS $=91.4 \mathrm{~mm})$.

The present crossover study also demonstrates the appropriateness of the titration-to-effect approach for opioid analgesics and the advantages of preparations that are available in a wide range of doses. A low proportion of patients remained on the lowest dose of BTDS $(5 \mu \mathrm{g} / \mathrm{h})$ for the duration of the active treatment period because, even at that dose, they reported a reduction in pain intensity from baseline. The majority of patients in the present study titrated to the highest available dose of $20 \mu \mathrm{g} / \mathrm{h}$ (based on pain control and side effects), and experienced a greater reduction in pain intensity $(35 \%)$ compared with those on the lowest dose of BTDS (27.7\%). These data reflect the safety and efficacy of the $5 \mu \mathrm{g} / \mathrm{h}$ to $20 \mu \mathrm{g} / \mathrm{h}$ dose range in patients titrated to effect weekly, with rescue analgesia as needed.

The change from baseline in mean pain scores during the first four days of the patch application were the same as the mean pain scores during the last three days of treatment, indicating that there was no end-of-dosing period failure with BTDS treatment.
In chronic pain, the goal of treatment is to maintain or restore function and improve quality of life (4). There were significant improvements from baseline for both treatments for secondary outcome measures, including the effect of pain on sleep, pain and disability (PDI and Quebec Back Pain Disability Scale) and the quality of life index (SF-36). Although the sample size of the present study was considered to be adequately powered for the primary metric of pain intensity outcomes, it was not powered to show a difference for the secondary outcomes, such as disability and quality of life, and as is common with many other comparative opioid studies, buprenorphine was not shown to be superior to placebo plus acetaminophen with codeine (rescue) for these secondary outcomes.

While the frequency of adverse events was higher for active BTDS than in the placebo group, the type of events reported were the same in both groups. There were no serious adverse events during active BTDS treatment. The most common events were typical opioid-related side effects. The overall mean severity of adverse events was the same for both groups. However, it should be noted that acetaminophen/codeine was permitted in both study arms so that during the active phase, BTDS was coadministered with acetaminophen/codeine, although at a lower average dose than during the placebo phase. Nausea and somnolence were the most common adverse events and occurred more frequently with BTDS. Interestingly, there was no difference between treatment groups for constipation - a side effect to which affected patients rarely develop tolerance while receiving opioid therapy. This finding is consistent with BTDS having a low propensity for constipation at these dose levels. During the open-label phase of the study, there was evidence of the development of tolerance to nausea (decreased from 38\% to $24 \%$ ) and somnolence (decreased from 30\% to $12 \%$ ), but not for constipation (27.4\% versus $28.6 \%$ ).

Patients reported local skin reactions at the site of patch application in both the active BTDS group (30.3\%) and in the placebo group (24.1\%). Most reactions were mild to moderate in severity, and occurred at the current patch site, rather than the previous site, indicating that the skin reactions are of short duration and subside shortly after removal of the patch. Because there are eight recommended sites for application of BTDS, patients were instructed to rotate the site of administration, and thus minimize the skin irritation in any one area.

Of those patients who completed all eight weeks of the random assignment phase, $82 \%$ chose to continue treatment with BTDS in an open-label evaluation, suggesting that BTDS was well tolerated and considered to be efficacious by the majority of the study population.

The results of the open-label extension of the present study demonstrate that BTDS is effective in the long-term management of chronic low back pain. The reductions in pain intensity and improvements in functional and quality of life assessments achieved during the randomized phase of the study in terms of change from baseline were sustained for treatment periods of up to six months. The mean final dose of BTDS in the open-label phase was comparable with the mean dose during the double-blinded phase, suggesting that there was maintenance of pain control without development of analgesic tolerance. Furthermore, $82 \%$ of patients reported receiving at least moderate benefit from BTDS in the open-label phase. 
The only other opioid available in a transdermal formulation in Canada is fentanyl. Transdermal fentanyl is indicated for the treatment of persistent moderate to severe chronic pain that is not adequately managed with short-acting or weak opioids or combination products, and only in patients who are already receiving opioid therapy at a total daily dose of at least $60 \mathrm{mg} /$ day of morphine (or analgesic equivalent). It is contraindicated in opioid-naive patients, even at its lowest dose. Conversely, in opioid-naive patients with moderate pain, BTDS did not elicit a distinctly different safety profile for serious untoward events compared with placebo plus acetaminophen/codeine (rescue). In the current study, 58\% of randomly assigned patients were not receiving opioids before the study and it might be anticipated that the response of such patients to BTDS would be greater than that of patients who were opioid tolerant. However, post hoc exploratory analysis showed that there were similar significant improvements from baseline pain in both opioid-naive and non-naive patients.

\section{REFERENCES}

1. Statistics Canada. Back pain. <http://www.statcan.gc.ca/pub/ 82-619-m/2006003/4053542-eng.htm > (Accessed on December 9, 2008).

2. Woolf AD, Pfledger B. Burden of major musculoskeletal conditions. Bull World Health Organ 2003;81:646-56.

3. Rossignol M, Arsenault B, Dionne C, et al. Clinic on Low-Back Pain in Interdisciplinary Practice (CLIP) guidelines. Montréal: Direction de santé publique, Agence de la santé et des services sociaux de Montréal, 2007.

4. Toward Optimized Practice. Management of low back pain. <http://www.topalbertadoctors.org/cpgs/back_pain.html> (Accessed on November 20, 2009).

5. van Tulder MW, Scholten RJ, Koes BW, Deyo RA. Nonsteroidal anti-inflammatory drugs for low back pain: A systematic review within the framework of the Cochrane Collaboration Back Review Group. Spine 2000;25:2501-13.

6. Dapas F, Hartman SF, Martinez L, et al. Baclofen for the treatment of acute low-back syndrome. A double-blind comparison with placebo. Spine 1985;10:345-9.

7. Arbus L, Fajadet B, Aubert D, Morre M, Goldberger E. Activity of tetrazepam (Myolastan R) in low back pain. A double-blind trial vs. placebo. Clin Trials J 1990;27:258-67.

8. Levy MH. Pharmacologic treatment of cancer pain. N Engl J Med 1996;335:1124-32.

9. Ofman JJ, MacLean HC, Straus WL, et al. A meta-analysis of severe upper gastrointestinal complications of nonsteroidal antiinflammatory drugs. J Rheumatol 2002;29:804-12.

10. McGettigan P, Henry D. Cardiovascular risk and inhibition of cyclooxygenase: A systematic review of the observational studies of selective and nonselective inhibitors of cyclooxygenase 2. JAMA 2006;296:1633-44.

11. Health Canada Advisory. Safety information regarding selective COX-2 inhibitor NSAIDs: Vioxx (rofecoxib), Celebrex (celecoxib), Bextra (valdecoxib), Mobicox (meloxicam) and generic forms of meloxicam, 2004. <http://www.hc-sc.gc.ca/ahc-asc/media/advisoriesavis/_2004/2004_69-eng.php> (Accessed on April 27, 2010).

12. Health Canada Advisory. Health Canada has asked Pfizer to suspend sales of its drug Bextra and informs Canadians of new restrictions on the use of Celebrex, 2005. <http://www.hc-sc.gc.ca/ ahc-asc/media/advisories-avis/_2005/2005_17-eng.php> (Accessed on April 27, 2010).

13. Antman EM, Bennett JS, Daugherty A, Furgberg C, Roberts H, Taubert KA. Use of nonsteroidal anti-inflammatory drugs. An update for Clinicians. A scientific statement from the American Heart Association. Circulation 2007;115:1634-42.

14. Goodkin K, Guillion CM. Antidepressants for the relief of chronic pain: Do they work? Ann Behav Med 1989;11:83-101.

15. Onghena P, Van Houdenhove B. Antidepressant-induced analgesia in chronic non-malignant pain: A meta-analysis of 39 placebocontrolled studies. Pain 2003;49:205-19.

\section{CONCLUSION}

Pain control was significantly better after four weeks of treatment with active BTDS than with placebo, even when active codeine plus acetaminophen was available to be taken as often as required. In both treatment groups, there was a significant improvement in pain scores from baseline levels. Six-month open-label treatment with buprenorphine demonstrated sustained analgesia, with increased tolerance to nausea and somnolence. BTDS provides a new treatment option for around-the-clock therapy in patients with moderate to severe low back pain.

ACKNOWLEDGEMENTS: The authors thank the late Dr John T Sibley MD (Royal University Hospital, Saskatoon, Saskatchewan) for his intellectual contributions and for the recruitment of patients for this study.

SUPPORT: Supported by a research grant from Purdue Pharma, Canada.

16. Atkinson JH, Slater MA, Wahlgren DR, et al. Effects of noradrenergic and serotonergic antidepressants on chronic low back pain intensity. Pain 1999;83:137-45.

17. Urquhart DM, Hoving JL, Assendelft WWJJ, Roland M, van Tulder MW. Antidepressants for non-specific low back pain (Review). Cochrane Database Syst Rev 2008;(1):CD001703.

18. Chou R, Fanciullo GJ, Fine PG, et al. American Pain Society opioid treatment guidelines: Clinical guidelines for the use of chronic opioid therapy in chronic noncancer pain. J Pain 2009;10:113-30.

19. Gourlay DL, Heit HA, Almahrezi A. Universal precautions in pain medicine: A rational approach to the treatment of chronic pain. Pain Med 2005;6:107-12.

20. Russo MA, Wasiak J. A clinical snapshot of transdermal buprenorphine in pain management. Eur J Pain 2007;1:74-7.

21. Kress HG. Clinical update on the pharmacology, efficacy and safety of transdermal buprenorphine. Eur J Pain 2009;13:219-30.

22. Dahan A, Yassen A, Romberg R, et al. Buprenorphine induces ceiling in respiratory depression but not in analgesia. $\mathrm{Br} \mathrm{J}$ Anaesth 2006;96:627-32.

23. Cowan A, Friderichs E, Strassburger W, Raffa RB. Basic pharmacology of buprenorphine. In: Budd K, Raffa R, eds. Buprenorphine - The Unique Opioid Analgesic. Stuttgart: Thieme Verlag KG, 2005:92-101.

24. Pergolizzi J, Boger RH, Budd K, et al. Opioids and the management of chronic severe pain in the elderly: Consensus statement of an international expert panel with focus on the six clinically most often used World Health Organization step III opioids (buprenorphine, fentanyl, hydromorphone, methadone, morphine, oxycodone). Pain Pract 2008;8:287-313.

25. Lewis JW. Clinical pharmacology of buprenorphine in relation to its use as an analgesic. In: Buprenorphine: Combatting Drug Abuse With a Unique Opioid. New York: Wiley-Liss Inc, 1995:151-63.

26. Kögel B, Christoph T, Strassburger W, Friderichs E. Interaction of $\mu$-opioid receptor agonists and antagonists with the analgesic effect of buprenorphine in mice. Eur J Pain 2005;9:599-611.

27. Christoph T, Kogel B, Schiene K, Meen M, De Vry J, Frederichs E. Broad analgesic profile of buprenorphine in rodent models of acute and chronic pain. Eur J Pharmacol 2005;507:87-98.

28. Karlsson M, Berggren AC. Efficacy and safety of low-dose transdermal buprenorphine patches $(5,10$, and $20 \mu \mathrm{g} / \mathrm{h})$ versus prolonged-release tramadol tablets $(75,100,150$, and $200 \mathrm{mg})$ in patients with chronic osteoarthritis pain: A 12-week, randomized, open label, controlled, parallel-group noninferiority study. Clin Ther 2009;31:503-13.

29. Landau CJ, Carr WD, Razzetti AJ, Sessler NE, Munera C, Ripa SR. Buprenorphine transdermal delivery system in adults with persistent noncancer-related pain syndromes who require opioid therapy: A multicentre, 5-week run-in and randomized, double-blind maintenance-of-analgesia study. Clin Ther 2007;29:2179-93. 
30. Pollard CA. Preliminary validity study for the pain disability index. Percept Mot Skills 1984;59:974.

31. Tait RC, Chibnall JT, Krause S. The Pain Disability Index: Psychometric properties. Pain 1990;40:171-82.

32. Kopec JA, Esdaile JM, Abrahamowicz M, et al. The Quebec Back Pain Disability Scale. Spine 1995;20:341-52.

33. Ware JE Jr, Sherbourne CD. The MOS 36-Item Short-Form Health Survey (SF-36). Conceptual framework and item selection. Medical Care 1992;30:473-83.

34. Farrar JT, Young JP Jr, LaMoreaux L, Werth JL, Poole RM. Clinical importance of changes in chronic pain intensity measured on an 11-point numerical pain rating scale. Pain 2001;94:149-58.
35. Farrar JT, Portenoy RK, Berlin JA, Kinman JL, Strom BL. Defining the clinically important difference in pain outcome measures. Pain 2000;88:287-94.

36. Moore A, McQuay H, Gavaghan D. Deriving dichotomous outcome measures from continuous data in randomized controlled trials of analgesics. Pain 1996;66:229-37.

37. Watson CP, Moulin D, Watt-Watson J, Gordon A, Eisenhoffer J. Controlled-release oxycodone relieves neuropathic pain: A randomized controlled trial in painful diabetic neuropathy. Pain 2003;105:71-8.

38. Sibley J, Kelly A, Rashiq S, et al. Controlled release oxycodone and acetaminophen plus codeine in chronic low back pain. In: Abstracts of the 10th World Congress on Pain. San Diego: International Association for the Study of Pain, 2002:429. 


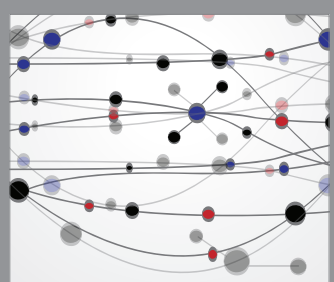

The Scientific World Journal
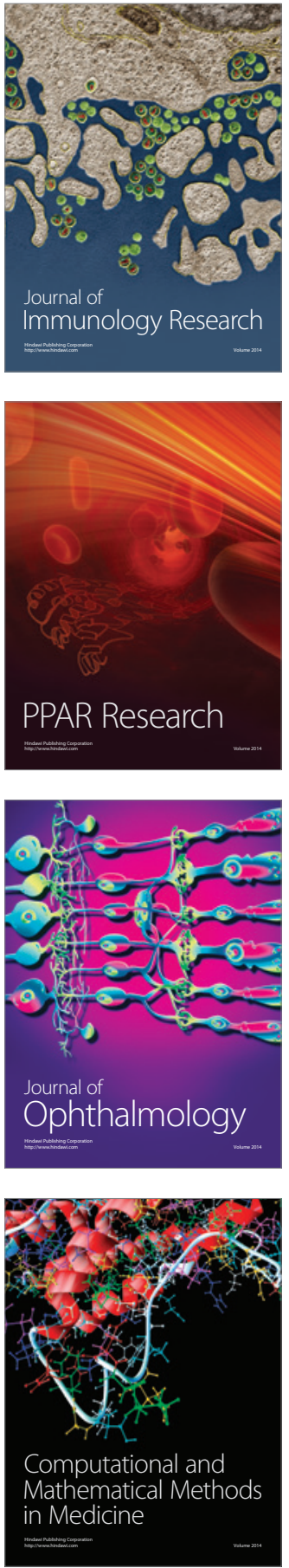

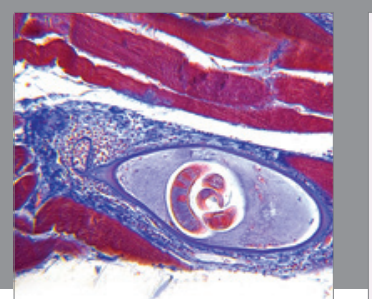

Gastroenterology Research and Practice

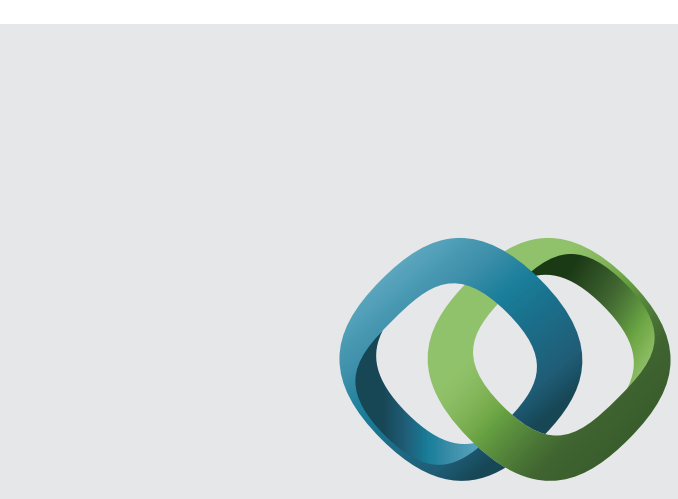

\section{Hindawi}

Submit your manuscripts at

http://www.hindawi.com
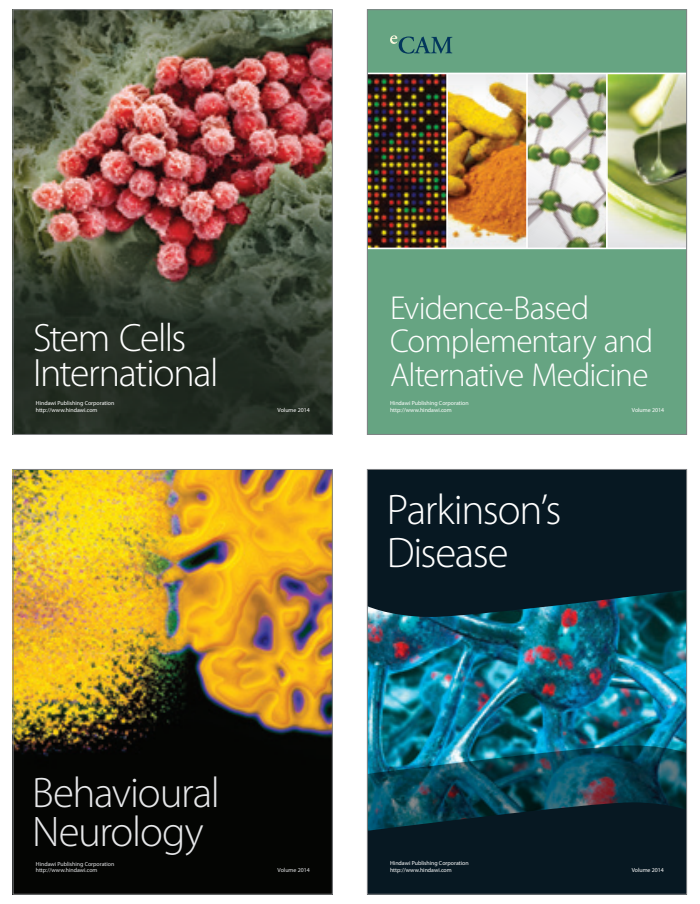
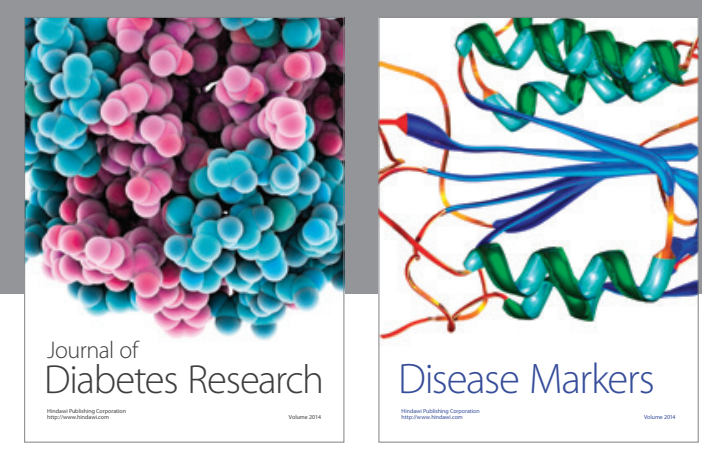

Disease Markers
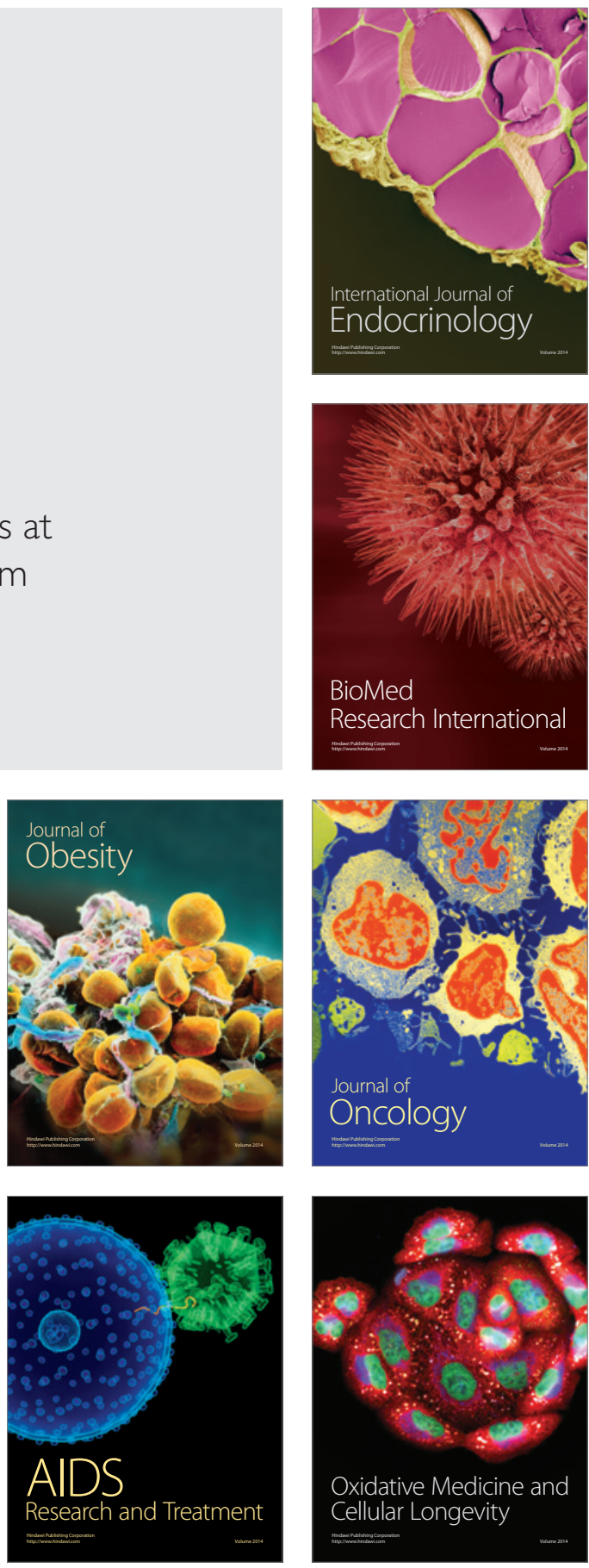